\title{
Cesarean Section in a COVID-19 Positive Patient with Takayasu Arteritis: A Case Report
}

\section{Ritesh Lamsal' • Navindra Raj Bista' • Utsav Acharya' • Pankaj Joshi' • Anil Shrestha'}

Abstract

Takayasu arteritis is an idiopathic, granulomatous aorto-arteritis that usually affects young or middle-aged women. The anesthetic management of patients with Takayasu arteritis undergoing cesarean section with COVID-19 is challenging. Patients may have concomitant hypertension, tissue hypoperfusion with the potential for major perioperative cardiovascular complications. The challenges increase considerably in resource-limited settings. We report the case of a 27-year-old primigravida at 39 weeks of gestation with concomitant Takayasu arteritis, chronic hypertension, hypothyroidism, and COVID-19, who underwent an elective cesarean section at our hospital. She incidentally tested positive for SARS-CoV-2 after admission to the hospital. The cesarean section was successfully performed under subarachnoid block. We are not aware of any previous publication describing the anesthetic management in patients with Takayasu arteritis and COVID-19 undergoing cesarean section.

Keywords: cesarean section; COVID-19; subarachnoid block; Takayasu arteritis

$\mathrm{T}$ akayasu arteritis is a large-vessel arteritis usually seen in women of child-bearing age. Optimal management of pregnant women with Takayasu arteritis, such as timing and method of delivery, is not clear. Anesthesiologists need to remain extremely vigilant as pregnant women with Takayasu arteritis can develop major perioperative cardiovascular complications. ${ }^{1}$ Major anesthetic challenges include choosing the most favorable type of anesthesia, anesthetic dosing, maintaining perioperative hemodynamics, and managing potential complications. ${ }^{2}$ The challenges multiply if patients also have coronavirus disease 2019 (COVID-19) infection. We describe the anesthetic management of a 27-year-old primigravida with Takayasu arteritis and COVID-19 undergoing cesarean section in a resource-limited setting. The patient tested positive for severe acute respiratory syndrome coronavirus 2 (SARS-CoV-2) incidentally, as all patients undergoing elective surgery are screened for COVID-19 before surgery according to the institutional protocol.

\section{CASE}

A 27-year-old primigravida (weight $66 \mathrm{~kg}$, height $162 \mathrm{~cm}$ )

\section{$\equiv \quad$ Anil Shrestha}

aanilsh@hotmail.com

1 Department of Anaesthesiology, Maharajgunj Medical Campus, Tribhuvan Universtiy, Kathmandu, Nepal presented to our hospital at 39 weeks of pregnancy. She was diagnosed with Takayasu arteritis five years back and was on corticosteroids, which were stopped during pregnancy. Detailed medical reports were not available. She also had hypertension and hypothyroidism for three years and was on amlodipine, metoprolol, and thyroxine. Laboratory results showed normal values of hemoglobin, leukocyte count, electrolytes, urea, creatinine, coagulation parameters, and thyroid function tests. An electrocardiogram showed evidence of left ventricular hypertrophy. COVID-19 reverse transcriptase-polymerase chain reaction was positive, even though she had no typical clinical features of recent infection. Echocardiography and Doppler scan of the arteries could not be obtained. Furthermore, there is no provision of a birthing center for COVID-19 patients in our hospital, so she was planned for an elective cesarean section in a COVID-19 dedicated operation room.

A formal pre-anesthetic check-up revealed that the patient could perform her normal daily activities and had no functional limitations. On examination, the heart rate was 110 beats per minute, and the blood pressure was $160 / 90 \mathrm{mmHg}$ and $160 / 80 \mathrm{mmHg}$ on the right and left arms, respectively. All peripheral pulses were palpable. On auscultation, bilateral carotid bruit was audible. The remaining systemic, obstetric, and fetal examinations were normal.

Standard intraoperative monitors consisted of a pulse oximeter, five-lead electrocardiogram, and non-invasive 
blood pressure. Two 18-G intravenous cannula were secured in the upper limbs, and a 20-G arterial catheter was inserted in the left radial artery. Before the subarachnoid block (SAB), preloading was done with $10 \mathrm{ml} / \mathrm{kg}$ of Ringer's lactate in 15 minutes. Under aseptic technique, the $\mathrm{SAB}$ was given at $\mathrm{L} 3 / \mathrm{L} 4$ level with $2.2 \mathrm{ml}$ of $0.5 \%$ hyperbaric bupivacaine using a 25-G Quincke spinal needle. After 10 minutes of the SAB, T6 sensory block was achieved. The lowest recorded blood pressure was 80/50 mmHg. As the patient had bilateral carotid bruit and there was no facility for neurological monitoring, we administered vasopressors to treat any drop of mean arterial pressure below 20\% of the baseline value. The patient received three boluses of mephentermine $6 \mathrm{mg}$ and one bolus of phenylephrine $50 \mu \mathrm{g}$ over 20 minutes. The rest of the intraoperative period was uneventful. The patient received a total of $1500 \mathrm{ml}$ of crystalloids intraoperatively. The blood loss was around $500 \mathrm{ml}$. The total duration of anesthesia was one hour. The newborn's APGAR scores were 7 and 10 at 1 and 5 minutes respectively. The patient was subsequently transferred to the COVID-19 intensive care unit for observation according to institutional protocol. The post-surgical recovery was unremarkable for both the mother and the baby.

\section{DISCUSSION}

Takayasu arteritis predominantly affects the aorta and its primary branches. The classification system proposed by the American College of Rheumatology is most commonly used for the diagnosis. ${ }^{3}$ The disease generally progresses insidiously, with patients exhibiting non-specific constitutional symptoms, like weight loss and fatigue in the early stages that sometimes culminate in serious vascular manifestations, like limb claudication, uncontrolled hypertension, visual impairment, myocardial infarction, and stroke. ${ }^{4}$ Takayasu arteritis worsens pregnancy outcomes and obstetric complications occur in nearly half of the patients. ${ }^{5}$ Mortality rates up to $21 \%$ have been reported in the peripartum period. ${ }^{6}$ As there is a dearth of large studies in pregnant patients with Takayasu arteritis, most of the perioperative management decisions are guided by anecdotal evidence gathered from case reports and series. We also do not know if there are any unique manifestations or cardiovascular interactions that require special attention in pregnant patients with Takayasu arteritis who have COVID-19.

It is difficult to assess disease activity in Takayasu arteritis, so imaging studies like angiography are useful to detect the extent and nature of vascular involvement.
This is particularly important since anti-inflammatory drugs may have been discontinued in pregnancy, as in our case. Multiple reports have highlighted the importance of monitoring disease progression, as Takayasu arteritis may intensify during pregnancy. ${ }^{7,8}$ However, this was not possible in our case. Invasive imaging examination, like conventional arteriography or CT-angiography, was not possible because of radiation risks. Furthermore, non-invasive tests like echocardiography and fetal ultrasonography were also not possible, as there was no separate diagnostic imaging facility for COVID-19 patients in our hospital at the time of writing this report.

The decision to choose the mode of delivery is mainly governed by the severity and extent of the disease. Vaginal delivery with epidural analgesia can be considered in patients with uncomplicated Takayasu's arteritis. Even though there is no reported difference between cesarean section and normal delivery in terms of maternal and fetal outcomes, vaginal delivery is theoretically better as it avoids the hemodynamic stress of surgery and anesthesia. ${ }^{9}$ However, this was not possible as there is no separate birthing center for patients with COVID-19 in our hospital.

In patients undergoing surgery, choosing regional or general anesthesia, intraoperative monitoring modalities, and other anesthetic decisions are usually at the discretion of the anesthesiologist as consensus guidelines do not exist. When the neuraxial technique is chosen, it is prudent to use a low-dose of a local anesthetic to minimize hemodynamic perturbations. Hyperbaric bupivacaine as low as $5 \mathrm{mg}$ (with fentanyl) and 7.5 mg (without fentanyl) have been described in patients with Takayasu arteritis undergoing cesarean section. ${ }^{10,11}$ However, we cautiously opted for a higher spinal anesthetic dose because of the seemingly uncomplicated vascular disease process in the patient along with our inclination to avoid conversion to general anesthesia in the backdrop of COVID-19. It is probably prudent to choose epidural or combined spinal-epidural anesthesia in patients with functional limitations or advanced cardiovascular complications.

Meticulous perioperative blood pressure control is essential in patients with Takayasu arteritis. A sustained drop in blood pressure can have a profoundly deleterious effect on organ perfusion that is already compromised because of the vascular pathology. An arterial catheter is useful irrespective of the type of anesthesia, as non-invasive blood pressure recording can be erroneous because of the vasculitis. There may also be discrepancies in the blood pressure values recorded in the two arms in Takayasu arteritis because of partial or complete occlusion of the subclavi- 
an, axillary, and brachial arteries. A vascular scan should be ideally performed before arterial cannulation but was not possible in our case as the ultrasound machine was not available inside the COVID-19 designated operation room.

Lastly, proper interdisciplinary coordination among anesthesiologists, obstetricians, cardiologists, and rheumatologists was difficult in this case because of limitations such as the availability of personal protective equipment, inadequate specialist services, and other equipment in COVID-19 designated areas of the hospital.

\section{CONCLUSION}

This report highlights the perioperative challenges faced by anesthesiologists in the current COVID-19 pandemic in resource-limited settings when managing complicated obstetric cases. Cesarean section in a COVID-19 patient with uncomplicated Takayasu arteritis can be successfully managed under spinal anesthesia.

\section{DECLARATIONS}

Ethics approval and consent to participate: Not applicable Consent for publication: Obtained from the patient.

Availability of data and materials: The datasets used and/ or analyzed during the current study are available from the corresponding author on reasonable request. All relevant data are within the manuscript and its supporting information files.

Competing interest: None

Funding: None

Authors' contributions: RL: conducted the case, concept, design, literature search, manuscript preparation, and manuscript review. NRB: concept, design, literature search, manuscript preparation, manuscript review, and final approval. UA: conducted the case, concept, literature search, manuscript review, and final approval. PJ: conducted the case, design, literature search, manuscript editing, and final approval. AS: concept, manuscript preparation, manuscript editing, and final approval. All authors have read and approved the final manuscript.

Acknowledgement: None

\section{REFERENCES}

1. Ishikawa K, Maetani S. Long-term outcome for 120 Japanese patients with Takayasu's disease. Clinical and statistical analyses of related prognostic factors. Circulation. 1994;90:1855-60.

2. Kathirvel S, Chavan S, Arya VK, Rehman I, Babu V, Malhotra $\mathrm{N}$, et al. Anesthetic management of patients with Takayasu's arteritis: A case series and review. Anesth An- alg. 2001;93:60-5

3. Arend WP, Michel BA, Bloch DA, Hunder GG, Calabrese LH, Edworthy SM, et al. The American College of Rheumatology 1990 criteria for the classification of Takayasu arteritis. Arthritis Rheum. 1990;33:1129-34.

4. Gornik HL, Creager MA. Aortitis. Circulation. 2008;117:3039-51.

5. Comarmond C, Mirault T, Biard L, Nizard J, Lambert M, Wechsler B, et al. Takayasu arteritis and pregnancy. Arthritis Rheumatol. 2015;67:3262-9.

6. Li J, Zhu M, Li M, Zheng W, Zhao J, Tian X, et al. Cause of death in Chinese Takayasu arteritis patients. Medicine (Baltimore). 2016;95:e4069.

7. Gatto $M$, laccarino L, Canova M, Zen M, Nalotto L, Ramonda $R$, et al. Pregnancy and vasculitis: A systematic review of the literature. Autoimmun Rev. 2012;11:A447-59.

8. Tanacan A, Unal C, Yucesoy HM, Duru SA, Beksac MS. Management and evaluation of pregnant women with Takayasu arteritis. Arch Gynecol Obstet. 2019;299:79-88.

9. Suri V, Aggarwal N, Keepanasseril A, Chopra S, Vijayvergiya R, Jain S. Pregnancy and Takayasu arteritis: A single centre experience from North India. J Obstet Gynaecol Res. 2010;36:519-24.

10. Gupta D, Arora N. Takayasu arteritis: Anaesthetic implications in caesarean section. J Clin Anesth. 2019;58:18-9.

11. Ioscovich A, Gislason R, Fadeev A, Grisaru-Granovsky S, Halpern S. Peripartum anesthetic management of patients with Takayasu's arteritis: Case series and review. Int J Obstet Anesth. 2008;17:358-64.

\section{How to Cite}

Lamsal R, Bista NR, Acharya U, Joshi P, Shrestha A. Cesarean section in a COVID-19 patient with Takayasu arteritis: a case report. JBPKIHS. 202O;3(2):38-4O. 